ARTICLE

https://doi.org/10.1038/s41467-019-10022-5

OPEN

\title{
Inhibition of MYC by the SMARCB1 tumor suppressor
}

April M. Weissmiller ${ }^{1}$, Jing Wang${ }^{2}$, Shelly L. Lorey ${ }^{1}$ Gregory C. Howard ${ }^{1}$, Ernest Martinez ${ }^{3}$, Qi Liu ${ }^{2} \&$ William P. Tansey (1) ${ }^{1}$

SMARCB1 encodes the SNF5 subunit of the SWI/SNF chromatin remodeler. SNF5 also interacts with the oncoprotein transcription factor MYC and is proposed to stimulate MYC activity. The concept that SNF5 is a coactivator for MYC, however, is at odds with its role as a tumor-suppressor, and with observations that loss of SNF5 leads to activation of MYC target genes. Here, we reexamine the relationship between MYC and SNF5 using biochemical and genome-wide approaches. We show that SNF5 inhibits the DNA-binding ability of MYC and impedes target gene recognition by MYC in cells. We further show that MYC regulation by SNF5 is separable from its role in chromatin remodeling, and that reintroduction of SNF5 into SMARCB1-null cells mimics the primary transcriptional effects of MYC inhibition. These observations reveal that SNF5 antagonizes MYC and provide a mechanism to explain how loss of SNF5 can drive malignancy.

\footnotetext{
${ }^{1}$ Department of Cell and Developmental Biology, Vanderbilt University School of Medicine, Nashville, TN 37232, USA. ${ }^{2}$ Center for Quantitative Sciences, Vanderbilt University School of Medicine, Nashville, TN 37232, USA. ${ }^{3}$ Department of Biochemistry, University of California at Riverside, Riverside, CA 92521, USA. Correspondence and requests for materials should be addressed to W.P.T. (email: william.p.tansey@vanderbilt.edu)
} 
S WI/SNF is a multi-subunit chromatin remodeling complex that is frequently mutated in cancer ${ }^{1}$. Roughly $20 \%$ of all human cancers carry mutations in a SWI/SNF component ${ }^{2}$, a frequency approaching that of the tumor-suppressor TP53 ${ }^{1}$. Although some tumor-associated alterations to SWI/SNF are gain of function and oncogenic ${ }^{3}$, the majority of mutations are loss of function ${ }^{4}$, pointing to a predominantly tumor-suppressive role for SWI/SNF complex members.

Perhaps the clearest example of a tumor-suppressive SWI/SNF protein is SNF5, a core component of the complex that is encoded by the SMARCB1 gene (also known as INI1 or BAF47) ${ }^{5}$. $S M A R C B 1$ is a bona-fide tumor suppressor 6,7 that is lost or inactivated in multiple malignancies, including malignant rhabdoid tumor $(\mathrm{MRT})^{8-10}$, which is an aggressive and often lethal pediatric cancer. Interestingly, loss or inactivation of SMARCB1 is the only recurring mutation in MRT - and often the only mutation detected in MRT genomes ${ }^{11}$ - pointing to expansive functions of SNF5 in tumor suppression. Loss of SNF5 in MRT compromises SWI/SNF integrity, causing widespread collapse of enhancers regulating differentiation, and mobilization of residual SWI/SNF complexes to super-enhancers essential for tumor cell maintenance ${ }^{12}$. Conversely, reintroduction of wild-type SNF5 into MRT cell lines induces cell cycle arrest, apoptosis, purging of aneuploid cells, and loss of tumorigenicity ${ }^{13-18}$, demonstrating that the absence of SNF5 remains a driving force in the malignant state of these cells. It is possible that the tumor-suppressive actions of SNF5 are exerted entirely through its role in chromatin remodeling, but given the breadth of impact of SNF5 on cancerrelevant processes, it is equally possible that SNF5 plays a multifaceted role in suppressing tumorigenesis.

In addition to functions within the SWI/SNF complex, SNF5 also binds to c-MYC ${ }^{19-21}$, an oncoprotein transcription factor with an extensive suite of protumorigenic activities ${ }^{22}$. SNF5 interacts directly with the carboxy-terminus of $\mathrm{MYC}^{19,21}$ and is proposed to stimulate the ability of MYC to transactivate its target genes ${ }^{19}$. The concept that SNF5 is a coactivator for MYC, however, conflicts with its well-established role as a tumor suppressor, with a report that SNF5 and MYC oppositely regulate a common set of genes ${ }^{21}$, with findings that loss of SNF5 in cancer is associated with activation of MYC target gene signatures ${ }^{8-10}$, and with recent observations that MYC inhibition can restrict rhabdoid tumor growth in vivo ${ }^{23}$. Given these disparities, it is clear that both the functional significance of the SNF5-MYC interaction-and the underlying mechanisms involved-are unresolved.

Here, we use a combination of biochemical and genomic approaches to interrogate how SNF5 impacts MYC. We demonstrate that SNF5 selectively inhibits the ability of MYC to bind DNA in vitro and in cells, and show that reintroduction of SNF5 into MRT cells results in a broad and comprehensive displacement of MYC from chromatin. By comparing SNF5 reintroduction with MYC inhibition, we further demonstrate that the actions of SNF5 on MYC are independent of its effects on chromatin remodeling, and instead are mediated via control of RNA-polymerase pause release at MYC-regulated genes. These observations show that SNF5 tempers target gene recognition by MYC, providing a mechanism to account for enhanced MYC function in MRT and suggesting that the tumor-suppressive functions of SNF5 are mediated, at least in part, by inhibiting MYC.

\section{Results}

SNF5 inhibits DNA binding by MYC. The carboxy-terminal basic helix-loop-helix leucine zipper (bHLHZip) region of MYC interacts with MAX to form a DNA-binding module that recognizes E-box DNA sequences (CACGTG) ${ }^{22}$. SNF5 binds within the bHLHZip, and although it has little if any effect on the MYC-MAX interaction ${ }^{21}$, the impact of SNF5 on the DNAbinding ability of full-length MYC:MAX heterodimers has not been determined.

First, we asked if SNF5 modulates DNA binding by MYC:MAX complexes in vitro. We reconstituted full-length MYC:MAX and MAX:MAX dimers from highly purified recombinant proteins ${ }^{24}$ (Supplementary Fig. 1a) and showed they specifically bind to Ebox-containing DNA in an electrophoretic mobility shift assay (EMSA; Supplementary Fig. 1b). We added recombinant SNF5 (Supplementary Fig. 1a) to these reactions, and observed that increasing amounts of SNF5 resulted in displacement of MYC: MAX complexes from DNA (Fig. 1a, compare lane 3 with lanes 4-7). This effect was specific to MYC:MAX complexes, as contaminating MAX:MAX dimers in these preparations were less sensitive to SNF5 addition, and purified MAX:MAX complexes were refractory to the effects of SNF5 (lanes 8-12). The impact of SNF5 in these assays was not a general result of binding to MYC, as addition of the MYC-interaction partner WDR5 $5^{25}$ did not disrupt DNA binding, but instead super-shifted MYC:MAX:DNA complexes (lane 1). Importantly, deletion of the conserved region of SNF5 containing two imperfect repeats-which mediate binding to $\mathrm{MYC}^{19}$-blocked SNF5-dependent displacement of MYC:MAX complexes from DNA (Fig. 1b, compare lanes 3 and 4 with lanes 5 and 6), showing that the ability of SNF5 to interact with MYC is required for disruption of DNA-binding. We conclude that SNF5 directly and selectively inhibits the DNAbinding ability of MYC in vitro.

Next, we asked if SNF5 modulates DNA binding by MYC in cells. We developed a system that allowed us to acutely manipulate SNF5 protein levels via the dTAG approach ${ }^{26}$. We knocked-out (KO) SMARCB1 via CRISPR-mediated genome editing in HEK293 cells, and then expressed a form of SNF5 that is tagged with FKBP12 $2^{(\mathrm{F} 36 \mathrm{~V})}$ (Supplementary Fig. 1c, d). In the presence of degrader dTAG-47 ${ }^{27}$, tagged SNF5 was destroyed rapidly (Fig. 1c). Indeed, $2 \mathrm{~h}$ of treatment with dTAG-47 was sufficient to deplete most SNF5, with little if any effect on MYC protein levels (Supplementary Fig. 1e). We then used chromatin immunoprecipitation (ChIP) to monitor MYC binding to nine MYC-bound loci ${ }^{28}$ under each condition. Here, we found that reexpression of SNF5 in KO cells reduced MYC binding to chromatin at most of the genes tested (with the exception of CCT7), and that this effect could be rapidly reversed by dTAG47-triggered degradation of SNF5 (Fig. 1d). Thus as we observed in vitro, SNF5 expression in cells can interfere with the ability of MYC to bind target genes in the context of chromatin.

SNF5 inhibits target gene recognition by MYC in MRT cells. To look at the impact of SNF5 on MYC in a more tumor-relevant setting, we asked whether SNF5 modulates the interaction of MYC with chromatin in the context of MRT, which is driven by SNF5 loss, and where MYC target gene signatures are repeatedly activated. To determine if MYC is important in MRT cells, we first attenuated its expression and asked how this alters MRT cell viability and anchorage-independent growth. Here, we observed that shRNA-mediated knockdown of MYC significantly decreased both parameters (Supplementary Fig. 2), confirming the importance of MYC to MRT cells and reinforcing the notion that this is an appropriate setting in which to interrogate the influence of SNF5 on MYC.

We next established a system that allowed us to compare the effects of reintroduction of SNF5 in MRT cells with inhibition of MYC in the same setting. G401, an extensively studied (and MYC-dependent; Supplementary Fig. 2) MRT cell line ${ }^{13-15,17,18,}$ 


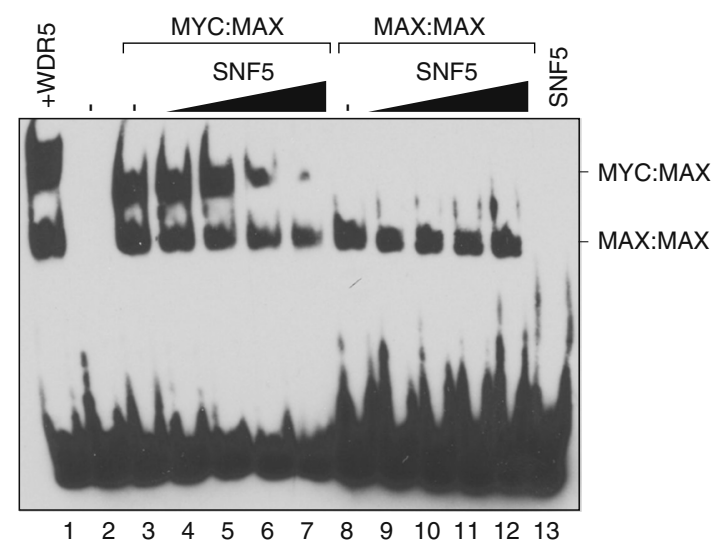

b

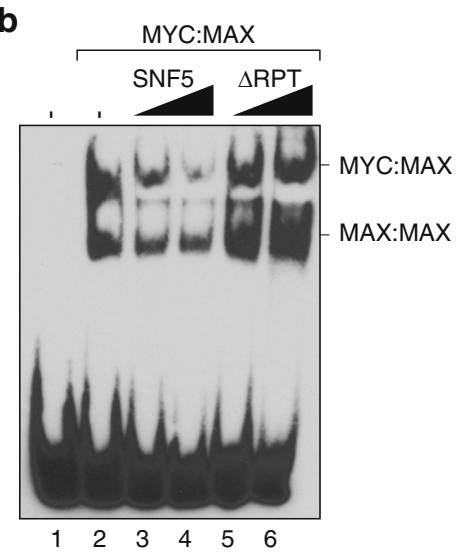

C

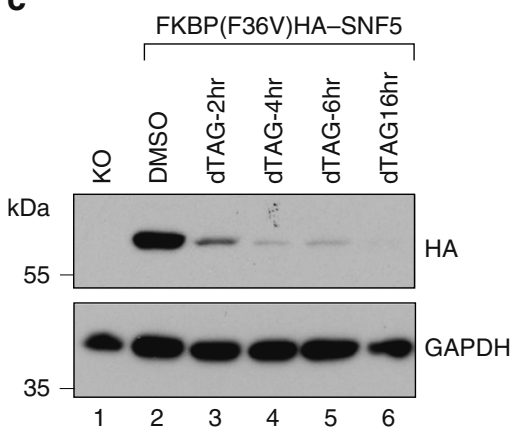

d

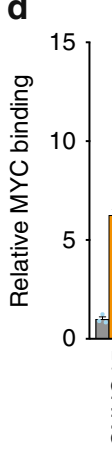

$\lg G$

$\square \mathrm{KO}$

+SNF5(DMSO)

+ SNF5(dTAG)

Fig. 1 SNF5 inhibits DNA-binding by MYC. a Recombinant MYC:MAX or MAX:MAX complexes were incubated with increasing amounts of recombinant SNF5 (3-, 6-, 12-, 24-fold molar excess over MYC:MAX) and EMSA performed using a DNA probe carrying a wild-type E-box sequence. Note that MYC: MAX complexes also contain residual MAX:MAX dimers. Lane 1 shows that incubation with the MYC interactor WDR5 (12-fold molar excess) induces a supershift in the MYC:MAX complexes. Lane 13 is the top concentration of SNF5, without MYC or MAX proteins. b EMSA, as in a, except MYC:MAX complexes were incubated with either recombinant SNF5 (12-, 24-fold molar excess) or an SNF5 mutant lacking the imperfect repeats region (amino acids 176-309) that interact with MYC ( $\triangle R P T ; 12-, 24-$ fold molar excess). Results were confirmed with two independent preparations of recombinant proteins.

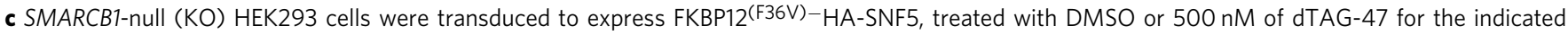
times, lysates prepared, and the levels of HA-tagged degradable SNF5 determined by immunoblotting. Lysate from the KO cells is included as a control in

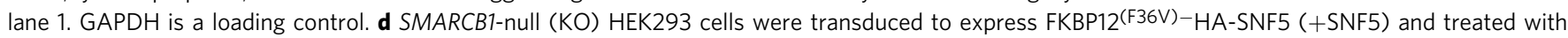
DMSO or dTAG-47 (500 nM) for $2 \mathrm{~h}$. ChIP assays were then performed. IgG control is shown for the dTAG-47-treated sample. Anti-MYC ChIPs are shown for the KO and the treated +SNF5 cells. Relative MYC binding is calculated as the signal at each of the indicated loci, relative to a non-MYC-bound locus ( $\beta$-globin). $n=3$ independent ChIP experiments. Error bars are standard error

was engineered to express inducible forms of enhanced green fluorescent protein (EGFP), SNF5, or OmoMYC-a dominantnegative mutant that blocks the productive association of MYC with its target genes ${ }^{29-32}$. In this experimental system the level of reintroduced SNF5 was comparable to endogenous SNF5 in other cell lines (Supplementary Fig. 3a). Reintroduced SNF5 interacted with the core SWI/SNF component BAF155 (Supplementary Fig. 3b), and co-migrated with other SWI/SNF complex subunit members in glycerol sedimentation assays (Supplementary Fig. 3c), consistent with its assembly into an SWI/SNF complex ${ }^{12}$. SNF5 also suppressed anchorage-independent growth of G401 cells in culture (Supplementary Fig. 3d, e). OmoMYC, as expected, reduced interaction of MYC with chromatin (Supplementary Fig. 3f). Notably, expression of SNF5 did not alter steady-state MYC protein levels (Supplementary Fig. 3g), providing the opportunity to look specifically at the effects of SNF5 on MYC function.

First, we coupled ChIP to next-generation sequencing (ChIPSeq) to track the distribution of MYC across the genome of G401 cells. We identified $~ 900$ peaks of MYC binding in G401 cells expressing the EGFP control (FDR of 0.01). This number of peaks was relatively low, compared to what has been reported for other cell types ${ }^{33}$, but the pattern of binding we observed was authentic for MYC. Binding sites were enriched in the E-box motif (Fig. 2a), predominantly promoter proximal (Fig. 2b), and enriched in genes linked to the well-established role of MYC in stimulating protein synthesis ${ }^{22}$ (Fig. 2c). Comparison with six published ChIP-Seq data sets revealed that more than half of the MYC binding sites we tracked in G401 cells are shared with the other six cell types (Fig. 2d), while a hypergeometric test showed significant overlap of our G401 data with the two MSigDB Hallmark MYC target gene collections (Fig. 2e), both of which contain different MYC target genes. Reducing the analysis FDR to a more relaxed value of 0.1 increased the number of MYC peaks to $\sim 1500$, but did not substantively change any of the relevant characteristics (Supplementary Fig. 4). We conclude that the number of MYC binding sites in G401 cells is comparatively low, but that those sites that are bound are strongly connected to the core functions of MYC.

We next compared EGFP with the effects of OmoMYC or SNF5 expression in G401 cells (Fig. 2f-i). Here, OmoMYC reduced detectable MYC binding genome wide, consistent with its known functions ${ }^{29-32}$. Importantly, SNF5 also reduced MYC binding. The effects of SNF5 on MYC were genome-wide and the average magnitude of reduction in binding intensity was about threefold (Fig. 2i). As described, steady-state levels of MYC are 
a

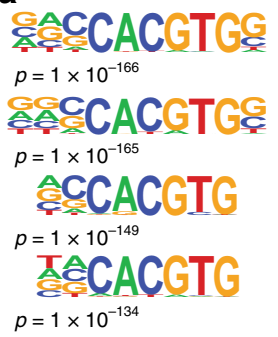

b

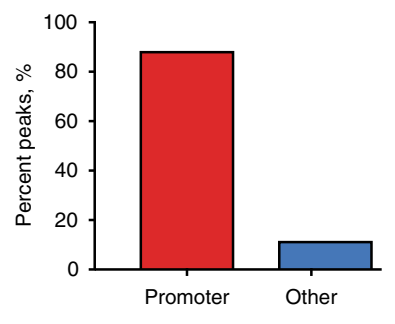

C

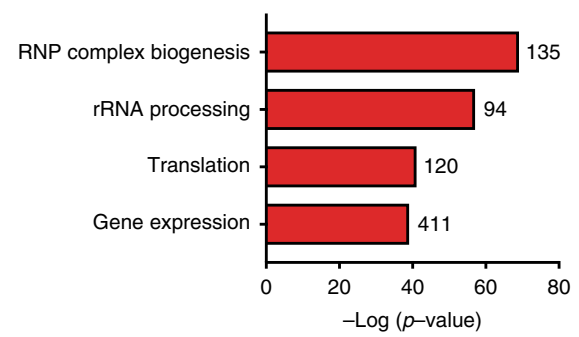

e
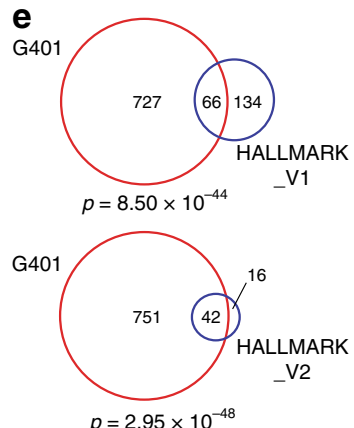

$p=2.95 \times 10^{-48}$

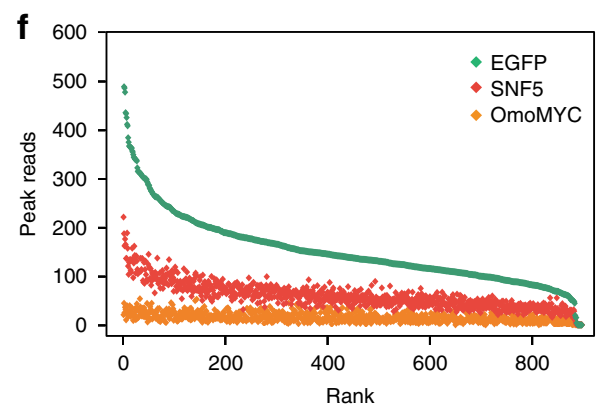

h

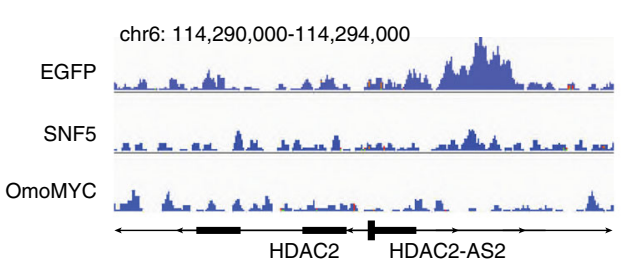

chr17: 35,304,000-35,308,000

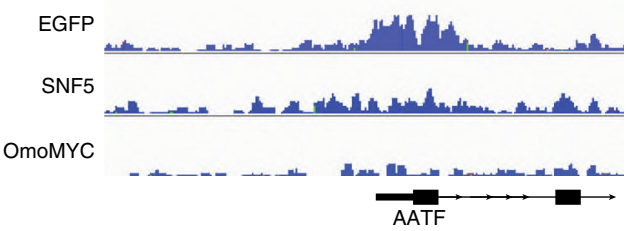

g EGFP

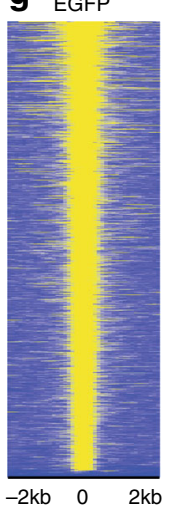

SNF5

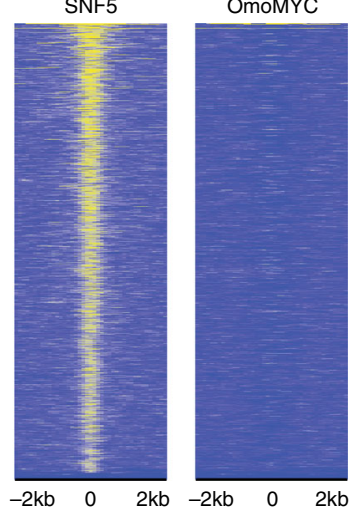

i

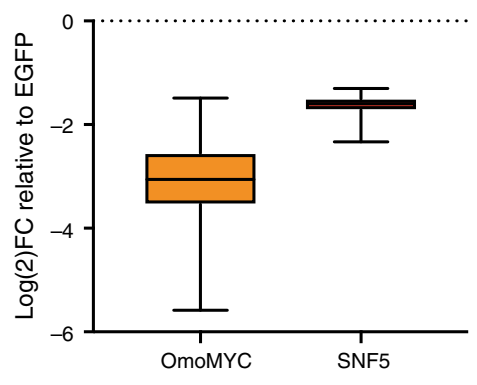

Fig. 2 SNF5 inhibits chromatin binding by MYC in MRT cells. a Known motif enrichment analysis was performed on the ChIP-Seq data from G401 cells expressing EGFP. The top four motifs are shown; all are enriched in the E-box sequence (CACGTG). b MYC peaks in the EGFP-expressing cells were quantified in terms of their distance to the nearest annotated transcriptional start-site (TSS). Peaks within $1 \mathrm{~kb}$ of a TSS are called as "promoter" peaks. c MYC peaks located within $1 \mathrm{~kb}$ from a TSS were assigned to their nearest gene and GO term enrichment analysis was performed. d Overlap of MYC peaks obtained from EGFP-expressing G401 cells with six published MYC ChIP-seq data sets: NB4 acute promyelocytic leukemia cells (GSM935643), hESC human embryonic stem cells (GSM935509), Ramos Burkitt's lymphoma cells (GSM762711), A549 lung cancer cells (GSM1003607), K562 chronic myeloid leukemia cells (GSM935516), and MCF7 breast cancer cells (GSM1006866). e MYC peaks that are located within $1 \mathrm{~kb}$ from a TSS were assigned to their nearest gene and overlaid with two MSigDB Hallmark MYC target data sets. A hypergeometric test was performed; significance is displayed below the Venn diagram. $\mathbf{f}$ Scatterplot of normalized peak read counts for each condition (average of replicates), ranked based on EGFP peak read number. $\mathbf{g}$ Heat maps of MYC peak intensity for each condition representing the combined average of normalized peak intensity in 100-bp bins $\pm 2 \mathrm{~kb}$ around the center of peaks. Genes ranked based on EGFP. $\mathbf{h}$ Two example genome browser tracks from each condition. $\mathbf{i}$ Box-and-whisker plot of log ${ }_{2}$-fold changes of $\mathrm{MYC}$ peaks for OMOMYC or SNF5 samples (FDR < 0.05), relative to EGFP. Box extends from 25th to 75th percentile with median marked by the middle line, whiskers extend from minimum to maximum point. $n=2$ independent ChIP-seq experiments 
a
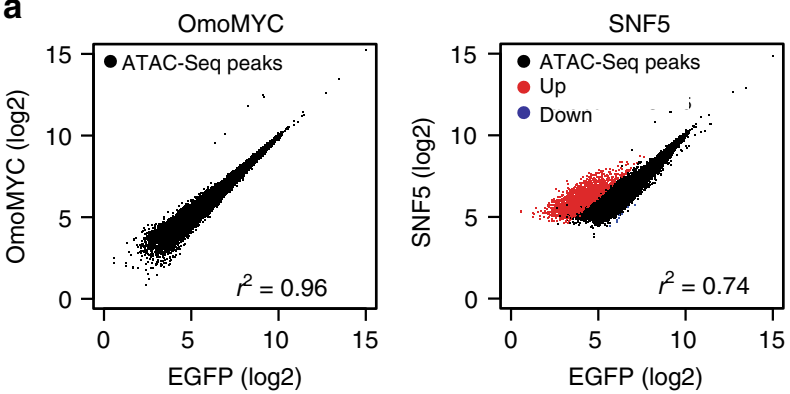

b

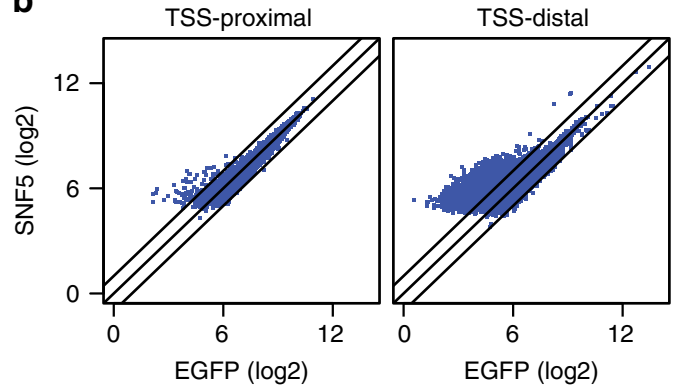

C

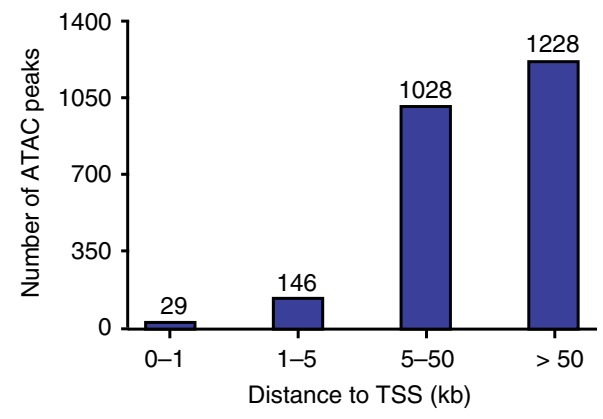

d Intracellular signal transduction Cell development Cell migration Localization of cell Reg. of multicellular development Cell proliferation Regulation of signaling Regulation of cell differientation Regulation of molecular function Angiogenesis

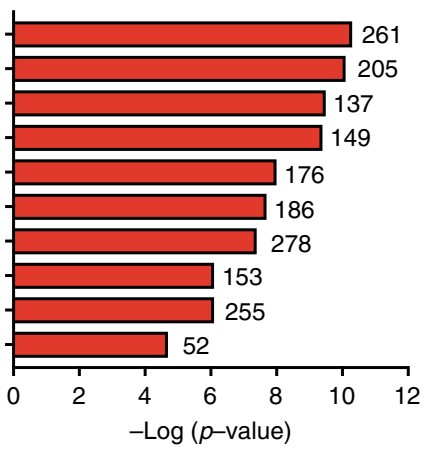

e

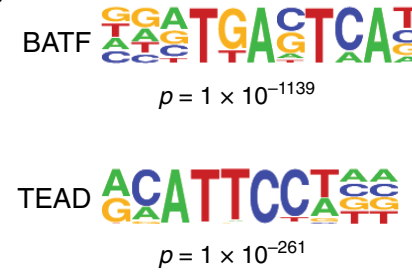

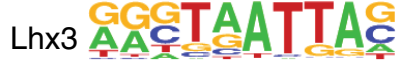

$p=1 \times 10^{-102}$

ZNF711

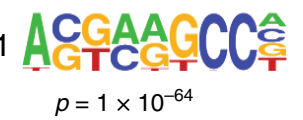

f

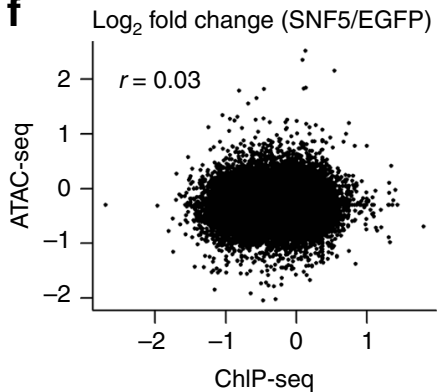

Fig. 3 SNF5 causes changes in chromatin state that are independent of its impact on MYC. a Scatterplots showing log 2 -fold changes in ATAC-Seq peaks for OmoMYC and SNF5, compared to EGFP samples. b Differential ATAC-Seq peaks were separated based on whether they are TSS-proximal ( $\leq 1 \mathrm{~kb}$ upstream or 100 bp downstream of the TSS) or TSS-distal (greater than these parameters). c Graph presents a breakdown of the 2491 ATAC-Seq peaks induced by SNF5, according to their distance from the nearest annotated TSS. d GO analysis of genes associated with ATAC-seq peaks induced by SNF5 expression. e De novo motif analysis of ATAC-seq peaks induced by SNF5 expression, showing the top four motifs identified. $\mathbf{f}$ Scatterplot showing the $\log _{2}$-fold changes of MYC ChIP-Seq read counts (SNF5/EGFP) on the $x$-axis and the $\log _{2}$-fold changes of ATAC-Seq read counts (SNF5/EGFP) on the $y$ axis at all promoters

unaffected by SNF5 expression at this same time point (Supplementary Fig. 3g), ruling out the possibility that the reduction in binding we observed is due to a decrease in MYC protein. These results are consistent with the response of MYC to altered SNF5 levels in HEK293 cells, and with our in vitro DNAbinding experiments, and demonstrate that SNF5 tempers the ability of MYC to bind chromatin in MRT cells.

Chromatin regulation by SNF5 is distinct from effects on MYC. Given that reintroduction of SNF5 into MRT cells will reconstitute SWI/SNF ${ }^{12}$, it is possible that the effects we observe on MYC binding in our G401 system are due to alterations in chromatin accessibility triggered by the SWI/SNF complex. To ask whether SNF5 alters the chromatin landscape at or around MYC target genes, we used the assay for transposase accessible chromatin followed by next-generation sequencing (ATAC-Seq) ${ }^{34}$ to identify changes in chromatin accessibility induced by EGFP, SNF5, and OmoMYC expression. Overall, we identified $\sim 25,000$ sites of open chromatin in the EGFP-expressing cells.
None of these sites were significantly affected by OmoMYC expression (Fig. 3a), indicating that displacement of MYC from its target genes in G401 cells does not substantively alter open chromatin status. In contrast, SNF5 expression resulted in a profound increase in chromatin accessibility, causing $\sim 2500$ new open chromatin sites to be formed (Fig. 3a). Only seven sites showed decreased accessibility upon SNF5 reintroduction (Fig. 3a). The majority of new open chromatin sites were transcription start site (TSS)-distal (Fig. 3b), with $90 \%$ being at least $5 \mathrm{~kb}$ from the nearest TSS, and half more than $50 \mathrm{~kb}$ away (Fig. 3c). Assignment of gained open chromatin peaks to their nearest gene showed a strong enrichment of genes involved in signal transduction, development, and differentiation (Fig. 3d). Moreover, gained sites are enriched for several DNA sequence motifs, including those belonging to the AP-1/ATF superfamily ${ }^{35}$ (Fig. 3e, Supplementary Table 1). These findings are consistent with the notion that SNF5 is important for enhancer regulation at critical cell identity genes $12,36,37$, and with published reports linking SNF5 to the AP-1/ATF proteins ${ }^{12,36,37}$. Importantly, the open chromatin peaks induced by SNF5 expression in G401 cells 
were entirely separable from MYC; there was no correlation between fold changes of MYC ChIP-Seq read counts (SNF5/ EGFP) and ATAC-Seq read counts (SNF5/EGFP) at all promoters (Fig. 3f, $r=0.03$ ), and no enrichment for E-box motifs in the ATAC-peak sequences (Supplementary Table 1). We conclude that reintroduction of SNF5 in G401 cells alters the chromatin landscape in a way that is consistent with its known functions, but that these effects are physically separable from its actions on MYC.

SNF5 inhibits RNA polymerase pause release at MYC targets. A key transcriptional function of MYC is to modulate release of paused RNA polymerases at its target genes ${ }^{38}$. To determine if the ability of SNF5 to temper MYC binding to chromatin impacts this activity, we used PRO-Seq ${ }^{39}$, a global nuclear run-on approach, to compare how SNF5 and OmoMYC alter the distribution of active RNA polymerases in G401 cells, genome-wide and at near-nucleotide level resolution. PRO-Seq also allowed us to follow primary transcriptional effects, and at the same time point $(24 \mathrm{~h}$ postinduction) as our ChIP- and ATAC-Seq experiments.

Compared to the EGFP control, OmoMYC and SNF5 induced a large number of transcriptional changes in the distribution of active RNA polymerases, both proximal to promoters and further inside gene bodies (Fig. 4a). Consistent with the role of MYC in promoting RNA polymerase pause release, OmoMYC increased the pausing index-the ratio of active polymerases at the promoter versus the gene body ${ }^{40}$-at $\sim 4500$ genes (Fig. $4 \mathrm{~b}$ ), with a smaller number $(2000)$ of genes showing a decrease in this ratio. SNF5, in contrast, produced an almost equal number of increases $(\sim 3500)$ and decreases $(\sim 3400)$ in pausing index. Notably, when we compared these two data sets, we found that $\sim 70 \%$ of the genes that show a change in pausing index with SNF5 are also changed with OmoMYC (Fig. 4c). Separating these genes according to the direction of change, we observed a highly significant correlation between the extent to which polymerase pausing was altered by OmoMYC and SNF5, both for genes showing a gain (Fig. 4d), as well as a loss (Fig. 4e), of pause. In total, $\sim 70 \%$ of genes gaining a pause with SNF5 also gained a pause with OmoMYC (Fig. 4f), and $\sim 35 \%$ of genes losing a pause with SNF5 lost a pause with OmoMYC (Fig. 4g). Principal component analysis of pausing indices revealed that the transcriptional effects of OmoMYC and SNF5 cluster more closely, and therefore have a similar effect, at MYC-bound, compared to MYC-unbound, genes (Supplementary Fig. 5a). And quantitative comparison of pausing index differences indicated that changes in pausing index were similar between OmoMYC and SNF5 at MYC-bound genes, but different at MYC-unbound loci (Supplementary Fig. 5b). Eighty percent of the MYC targets that gain a pause with SNF5 gain a pause with OmoMYC (Fig. 4h), a significantly higher level of overlap than for non-MYC targets (Supplementary Fig. 6a). In contrast, only 35\% of MYC target genes experience a loss of pause under both conditions (Supplementary Fig. 6b). In general, the extent and significance of overlap between SNF5 and OmoMYC was higher for genes experiencing RNA polymerase pause induction, and the types of genes regulated in each direction were different (Supplementary Fig. $6 c, d$ ), with pause-induced genes being enriched in those connected to canonical MYC functions, including protein synthesis. Based on these data, we conclude that reintroduction of SNF5 in G401 cells mimics many of the transcriptional effects of MYC inhibition, and that a major impact of SNF5 on transcriptional events is to promote pausing of RNA polymerase at genes regulated by MYC.

Finally, we asked if these changes in RNA polymerase distribution correlate with the known effects of SNF5 reintroduction on the transcriptome of G401 cells. Comparing our PRO-Seq data to published RNA-Sequencing (RNA-Seq) data sets gathered at 3 or 7 days after reconstitution of G401 cells with SNF5 $^{37}$, we observed highly significant correlations between the two: At day 3, $\sim 30 \%$ of the genes that gain a pause with both SNF5 and OmoMYC in our experiments showed reduced RNA levels by RNA-Seq (Supplementary Fig. 6e), and at day 7 this overlap was $40 \%$ (Supplementary Fig. 6 ). In contrast, there was no significant overlap between genes that lose a pause and those showing an increase in RNA levels at either time point (Supplementary Fig. 6g, h). The correlation between our PROSeq data and these published RNA-Seq data strongly implies that the ability of SNF5 to induce RNA polymerase pausing at genes regulated by MYC represents a significant mechanism through which it shapes the transcriptome.

\section{Discussion}

Given the frequency with which SWI/SNF components are perturbed in malignancy ${ }^{2}$, understanding the mechanisms through which alterations in SWI/SNF drive tumorigenesis is fundamental to understanding how many cancers form and how they can be treated. Among SWI/SNF-mutant cancers, those defined by loss of SNF5 are particularly intriguing. On one hand, these cancers have an unusually simple genetic profile, with a single driver mutation-loss of SMARCB1/SNF5-and little if any evidence of collaborating oncogenic events. On the other hand ${ }^{11}$, these cancers are early onset malignancies ${ }^{41}$ that are difficult to treat and most often lethal. The contrast between the genetic simplicity of cancers like MRT and their aggressive nature implies that loss of SNF5 leads to a multitude of pro-oncogenic effects. Here, we provide evidence that one part of tumor suppression by SNF5 is to temper MYC binding to DNA. The direct connection between SNF5 and MYC explains the recurring activation of MYC target gene signatures in $\mathrm{MRT}^{8-10}$ and, because of the broad suite of oncogenic activities possessed by $\mathrm{MYC}^{22}$, can help rationalize how loss of a single tumor suppressor can have such profound effects on cellular pathophysiology.

The evidence that SNF5 directly impedes DNA binding by MYC is compelling (Fig. 1), and in line with recent NMR-based studies showing that the imperfect repeats of SNF5-which are required for this activity (Fig. 1b) - recognize the DNA-binding surface of the MYC:MAX bHLHZip heterodimer in a manner that is mutually exclusive with DNA recognition ${ }^{42}$. What we do not know, however, is the biochemical context in which SNF5 tempers MYC in cells. We see (Supplementary Fig. 3c), as others have reported ${ }^{12,37}$, that SNF5 that is reintroduced into MRT cells is incorporated into an intact SWI/SNF complex, suggesting that there is little unincorporated SNF5 in our experiments. We also see in these experiments changes in chromatin accessibility (Fig. 3) that are consistent with functional reconstitution of SWI/ SNF. We cannot, however, exclude the possibility that there is some low level of free SNF5 that inhibits MYC binding, or that SWI/SNF transiently donates SNF5 for MYC inhibition. Regardless of the context, however, the ability of SNF5 to interfere with target recognition by MYC in vitro and in two different cellular systems demonstrates a clear biochemical mechanism through which SNF5 antagonizes a key MYC activity.

The data presented here show that reintroduction of SNF5 into MRT cells leads to changes in TSS-distal chromatin accessibility at sites connected to differentiation and development (Fig. 3), very much in line with recently documented functions of SNF5 in maintaining lineage-specific enhancers and cell identity ${ }^{12,36,37}$ and activating bivalent promoters at developmentally important genes $^{37}$. By comparing these activities with the location of MYC in G401 MRT cells, we show that SNF5 has a second set of 
a

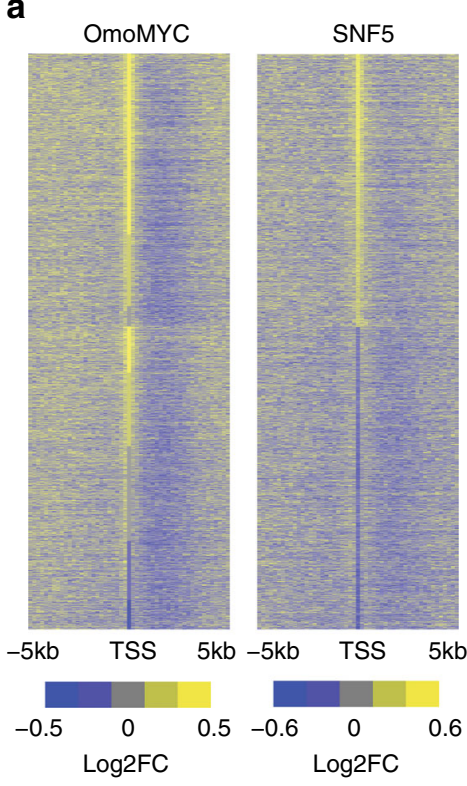

b

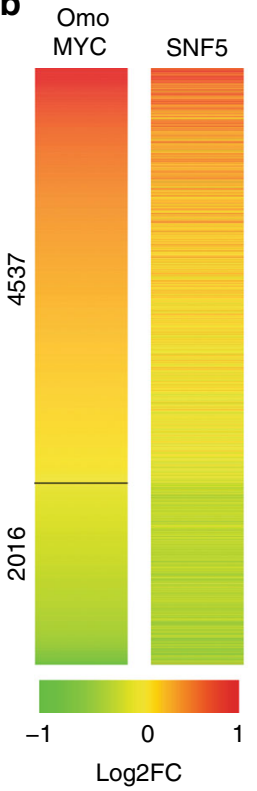

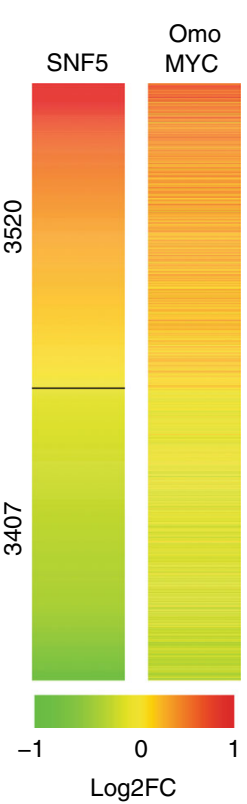

C

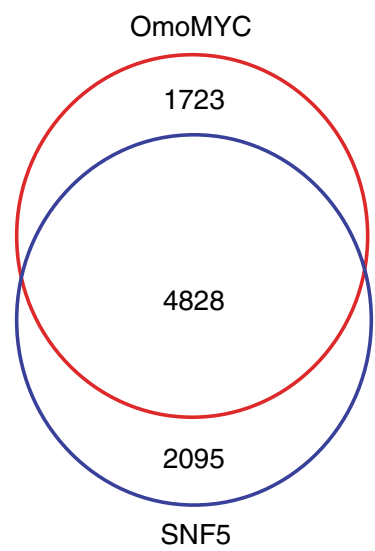

$p<10^{-308}$ d

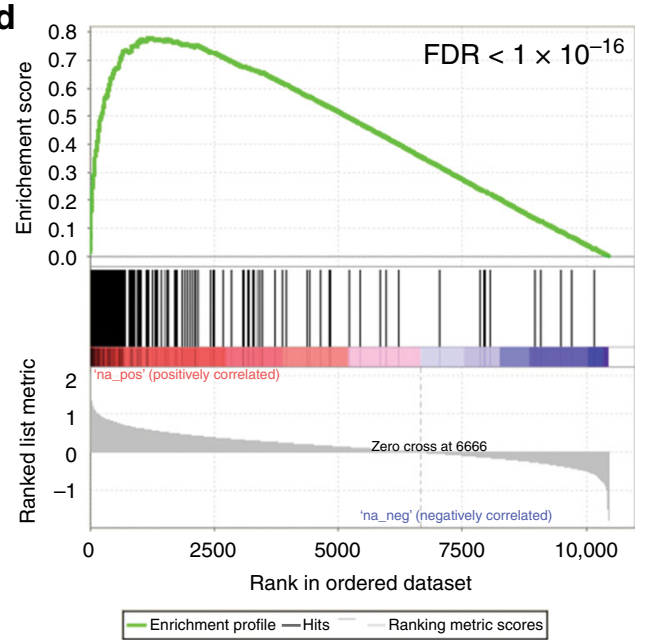

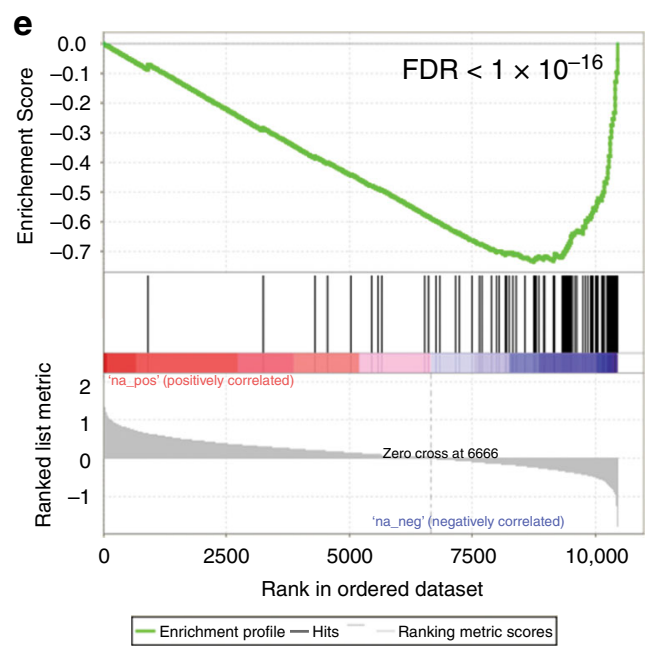

$\mathbf{f}$

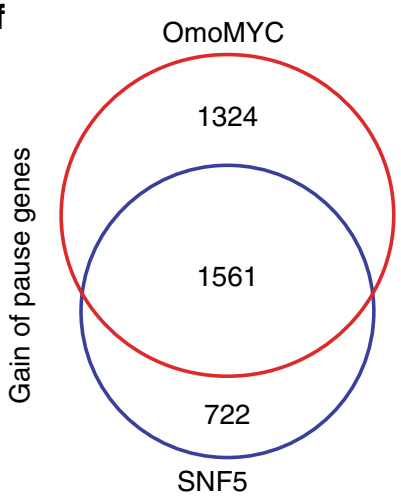

$p<10^{-308}$ g OmoMYc

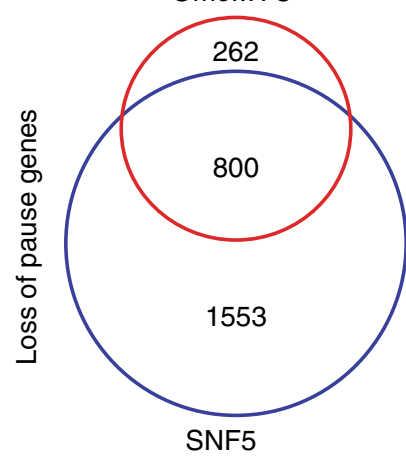

$p<10^{-308}$ h

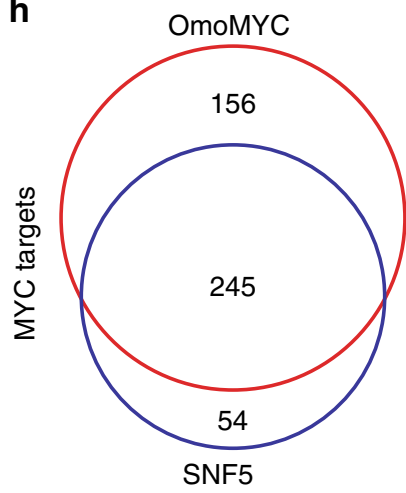

$p<10^{-308}$ activities-against MYC-that are almost exclusively promoterproximal (Fig. 2), and induce RNA polymerase pause arrest at genes regulated by MYC (Fig. 4). Our ability to physically separate the canonical functions of SNF5 from its anti-MYC activities reveals that SNF5 does not modulate MYC binding via changes in chromatin accessibility, and supports a revised model in which dual regulation of both chromatin accessibility (at TSS-distal enhancers) and control of MYC (at TSS-proximal promoters) are part of the SNF5 tumor-suppression program (Fig. 5).

What is the functional significance of a dual tumorsuppression mechanism for SNF5? In cells such as G401, which possess a modest number of MYC binding sites, almost all of which are promoter-proximal (Fig. 2), these two mechanisms would impact distinct sites in the genome, allowing SNF5 to 
Fig. 4 SNF5 mimics the primary transcriptional effects of MYC inhibition. a Heatmaps displaying $\log _{2}$-fold change (Log2FC) of active polymerases (compared to EGFP) in the promoter-proximal region and $\pm 5 \mathrm{~Kb}$ around the transcription start site (TSS), as determined by PRO-Seq. The two heatmaps are sorted in the same order; genes with increase in both OmoMYC and SNF5, genes with increase in OmoMYC only, and other active genes. Genes in each group are ranked by the OmoMYC FC near the TSS. b Heatmaps of genes showing changes in pausing index for OmoMYC and SNF5, compared to EGFP. Left panel: genes are ranked based on $\log _{2}$-fold change of pausing index in OmoMYC, and the corresponding changes in SNF5 are shown on the right side. Right panel: genes are ranked based on $\log _{2}$-fold change of pausing index in SNF5, and the corresponding changes in OmoMYC are shown on the right side. c Overlap of unique genes that had a change in pausing index upon OmoMYC expression with those that had a change following expression of SNF5 (FDR < 0.05). d Gene set enrichment analysis showing the distribution of genes that had an induced pause following SNF5 reintroduction against the ranked list of all genes with change in pausing index following OmoMYC expression (FDR $<0.05$, genes ranked by $\log _{2}$-fold change). e Gene set enrichment analysis, as in d, except for genes that had loss of pause following SNF5 reintroduction. $\mathbf{f}$ Overlap of genes that had increased pausing index (induced pause) for both OmoMYC and SNF5 (FDR < 0.0001). g Overlap of genes that had decreased pausing index (pause release) for both OmoMYC and SNF5 (FDR < 0.0001). h Overlap between the number of genes identified as MYC targets from ChIP-seq analysis (Fig. 3) that also had a resulting induced pause for each condition. $n=3$ independent PRO-Seq experiments

a

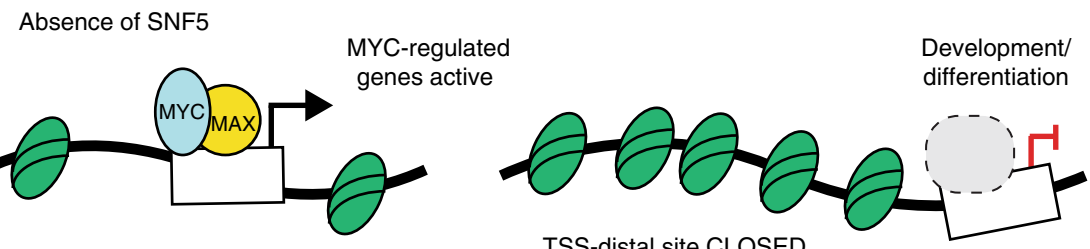

TSS-distal site CLOSED

b

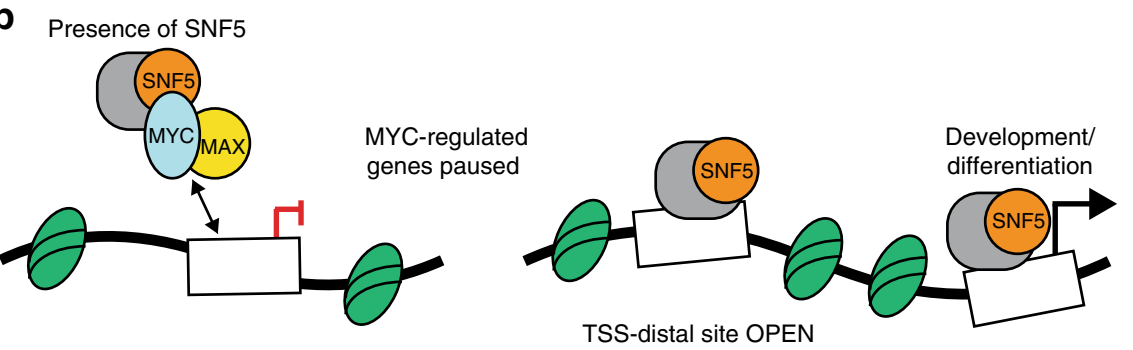

Fig. 5 Model: Dual tumor-suppressor activities of SNF5. a In the absence of SNF5, MYC binds unimpeded to chromatin to promote gene expression programs that maintain the MRT state. Under these conditions, transcription start site (TSS)-distal sites that regulate transcription of genes involved in development and differentiation remain in a closed state, repressing transcription. $\mathbf{b}$ When SNF5 is present, MYC binding to DNA is tempered and RNA polymerase pauses at these genes, repressing transcription. SNF5, in concert with the SWI/SNF complex (gray), also leads to remodeling at TSS-distal sites, resulting in an open chromatin state and activation of genes linked to development and differentiation

support both the broad transcriptional patterning achieved by enhancer/super-enhancer regulation, as well as the gene-specific control that can be achieved via targeting a sequence-specific transcription factor such as MYC. By directly modulating MYC at promoters, SNF5 would have the ability to couple enhancermediated cell identity determination with essential ancillary processes such as regulation of the cell cycle and biomass production (Supplementary Fig. 6c); an activity that would be critical during processes such as development-where SNF5 plays a key role in maintaining the balance between pluripotency and differentiation ${ }^{43}$-but upon SNF5 loss could readily conspire to drive tumorigenesis. Indeed, although the promoter-specific function of SNF5 in MRT has not previously been reported, our PRO-Seq studies clearly show that SNF5 impacts primary transcriptional events at MYC target genes in G401 cells and mimics MYC inhibition. Many of these changes in polymerase distribution correlate with changes in transcript levels, implying that the changes we see are relevant to disease processes in MRT. We also point out that the separable functions of SNF5 in regulating chromatin structure and tempering MYC do not necessarily have to operate at distinct regions of the genome. High levels of MYC overexpression lead to broad invasion of promoters as well as enhancers by $\mathrm{MYC}^{44}$, and are associated with MYC binding to degenerate E-box elements. In cells with a high MYC burden, therefore, MYC that is bound at enhancers may be particularly sensitive to inhibition, and the tumor-suppressive functions of SNF5 could act within the context of SWI/SNF to both maintain normal patterns of open chromatin status at key enhancers and to resist cooption of enhancer function by ectopic MYC.

One of the challenges in treating cancers such as MRT is that loss of a key tumor suppressor such as SNF5 does not readily reveal a strategy where drug-like molecules-most of which are inhibitors-could be effective. Most children diagnosed with MRT die quickly from highly metastatic disease, despite treatment regimens that can involve combinations of surgery, chemotherapy, and radiation. Some modest improvements in patient survival have been made in recent years ${ }^{45}$, but there is currently no standard of care for treating MRT sufferers, and the chances that a child diagnosed with MRT will survive a year, let alone 5 years, is very small ${ }^{45}$. Our data strongly imply that loss of SNF5 drives MRT, in part, by derepressing MYC. Although there are no drug-like MYC inhibitors available at present, the importance of MYC to human cancer fuels intense interest in their discovery, and a variety of direct and indirect methods to target MYC in the clinic are being moved forward ${ }^{46}$. Many of these approaches target the interaction of MYC with chromatin. OmoMYC itself is being developed in this capacity ${ }^{46}$, and has in vivo action against atypical teratoid rhabdoid tumors ${ }^{23}$, which (like MRT) are caused by SNF5 loss. The striking parallels we see between SNF5 
reintroduction and OmoMYC expression in G401 cells-as well as our finding that MRT cell lines depend on MYC for viabilitylays a strong conceptual foundation for the idea that MYC inhibition would be unexpectedly effective in treating this malignancy, and others driven through inactivation of SNF5.

\section{Methods}

Cell culture and transductions. G401, A204, and HEK293 cell lines were obtained from ATCC and maintained in Dulbecco's Modified Eagle Medium (DMEM) supplemented with $10 \%$ FBS and 1\% penicillin/streptomycin, or RPMI with $10 \%$ fetal bovine serum (FBS) and 1\% penicillin/streptomycin, respectively. HEK293 cells were maintained in DMEM supplemented with $10 \%$ FBS and $1 \%$ penicillin/ streptomycin. All cell lines used were confirmed as mycoplasma-negative. For inducible constructs, lentiviral transductions were performed by transfecting HEK293 cells with the appropriate inducible construct, the pMD2.G envelope expressing plasmid, and the psPAX2 packaging plasmid, which were gifts from Didier Trono (Addgene plasmid \#12259 and \#12260, respectively). Viral supernatant was collected in DMEM with 10\% TET-system approved FBS (Clontech 631106) and 1\% penicillin/streptomycin, and used to infect G401 cells. Stable cells were selected for 6 days using $0.25 \mathrm{mg} / \mathrm{ml} \mathrm{G418}$ in DMEM with 10\% TET-systemapproved FBS and 1\% penicillin/streptomycin. After selection, cells were counted, treated with $1 \mu \mathrm{g} / \mathrm{ml}$ doxycycline (Sigma D9891) for $24 \mathrm{~h}$, and experiments performed as described below. For shRNA constructs, transductions were performed as described, but viral supernatant was collected in normal FBS maintenance media and used to infect G401 and A204 cells. Cells were transduced for 2 days with shRNA viral supernatants, allowed to recover 1 day, and then a portion of the cells were counted on day 3 , and at each time point, by flow cytometry as described below. For dTag experiments, transductions were performed as described above with normal FBS maintenance media and those viral supernatants were used to infect an HEK293-SNF5-KO clone. Cells were transduced for 2 days and expanded for an additional 4-6 days before plating for ChIP experiments as described below.

Generation of SMARCB1-knockout cell lines. HEK293 cells were transfected with the Inil CRISPR/Cas9 KO Plasmid (Santa Cruz Biotechnology, sc-401485) and Ini1 HDR Plasmid (Santa Cruz Biotechnology, sc-401485-HDR) using Lipofectamine 3000 (Life Technologies), following the manufacturer's protocol. After $72 \mathrm{~h}$, cells were plated into a large dish with puromycin to allow for individual colonies to form. Individual colonies representing single clones were amplified and screened for the presence of SNF5 using two independent antibodies to identify SNF5-null cell lines.

Flow cytometry analysis. G401 and A204 cells transduced with appropriate shRNA vectors were collected and resuspended in DMEM with no phenol red. $2.5-5 \times 10^{5}$ cells were filtered through a $35 \mu \mathrm{m}$ nylon mesh Falcon round bottom test tube and the remaining cells returned to culture until the next time point. Filtered cells were delivered to the Vanderbilt Flow Cytometry Shared Resource for analysis of the number of GFP-positive cells using a Becton Dickinson LSRFortessa instrument. For each time point, 20,000 cells were counted using forward and side scatter to select single cells. The number of GFP-positive cells in the population was expressed as a percent of single, nonaggregated, cells. Initial experiments performed with addition of propidium iodide confirmed little to no toxicity of shRNA expression in GFP-positive cells. The gating strategy used for flow cytometry experiments is shown in Supplementary Fig. 7.

Creation of lentiviral constructs. Lentiviral shRNA constructs were designed by VectorBuilder (Cyagen) to include either a scrambled shRNA or one of two shRNAs against human MYC shRNA (from their database), driven by the U6 promoter. Each construct also contained an EGFP:T2A:Puromycin cassette driven by the PGK promoter. All lentiviral shRNA constructs were validated in HEK293 cells after selection with puromycin to confirm knockdown of MYC. Tet-inducible lentiviral vector OMOMYC was created by introducing four mutations ${ }^{29}$ into the DNA-binding domain of MYC by site-directed mutagenesis. OMOMYC was then PCR amplified and inserted into the multiple cloning site of pENTR1A ${ }^{47}$, with sequences encoding an HA-epitope tag, via Gibson assembly. pENTR1A (no ccDB (w48-1)) plasmid was a gift from Eric Campeau \& Paul Kaufman (Addgene plasmid \#17398). EGFP was amplified from the pEGFP-N1 plasmid (Clontech) and SNF5 was amplified from the pFastBacl INI1 construct, which was a gift from Robert Kingston (Addgene plasmid \#1953); both were inserted into the pENTR1A plasmid with sequences encoding an HA-epitope tag. Gateway cloning was used to insert each pENTR1A fragment into the lentiviral pInducer 20 acceptor vector ${ }^{48}$, which was a gift from Stephen Elledge (Addgene plasmid \#44012). To create a degradable SNF5 construct, the pENTR1A fragment containing SNF5 was gateway cloned in frame into the lentiviral pLEX-305-N-dTAG, a gift from James Bradner (Addgene plasmid $\# 91797)^{26}$. All plasmid constructions were confirmed by DNA sequencing.
Electrophoretic mobility shift assays. SNF5 coding sequences were amplified from the pFASTBacl INI1 construct and inserted into a pSUMO expression vector $^{25}$, carrying an N-terminal $6 \times$-HIS tag for purification. SUMO-WDR5 ${ }^{25}$, SUMO-SNF5, SUMO-SNF5 $\triangle$ RPT, and a control SUMO construct encoding amino acids 533-580 of BAF155, were expressed in Rosetta E. coli cells (EMD Millipore) and purified using a nondenaturing lysis buffer and Ni-NTA agarose, according to the Ni-NTA QIAGEN protocol (Qiagen). SUMO-SNF5 $\triangle$ RPT was engineered by deleting amino acids 176-309 in SUMO-SNF5 using Q5 whole-plasmid mutagenesis (NEB). Recombinant $6 \times$-HIS MYC and MAX were expressed in Rosetta E. coli cells and purified as previously published ${ }^{24}$. Functional MYC:MAX heterodimers were formed by combining a 3:1 molar ratio of MYC to MAX and performing step-wise dialysis ${ }^{24}$. DNA-binding reactions were performed using a double-stranded E box oligonucleotide bearing a 3'-biotin group: 5'-GCTCAGG GACCACGTGGTCGGGGATC-3' (IDT). The mutant E box oligonucleotide two changes ( $5^{\prime}$-GCTCAGGGACCAGCTGGTCGGGGATC- $3^{\prime}$ ). Binding reactions were performed in a 10-15 $\mu \mathrm{l}$ final volume and contained $15 \mathrm{mM}$ Tris- $\mathrm{HCl}(\mathrm{pH}$ 7.9), $15 \%$ glycerol, $100 \mathrm{mM} \mathrm{KCl}, 0.15 \mathrm{mM}$ ethylenediaminetetraacetic acid (EDTA), $0.075 \% \mathrm{NP}-40(\mathrm{v} / \mathrm{v}), 7.5 \mathrm{mM}$ 2-mercaptoethanol, $40 \mathrm{ng}$ poly $(\mathrm{dI}-\mathrm{dC}), 375 \mathrm{ng} / \mu \mathrm{l} \mathrm{BSA}$ and $25 \mathrm{fmol}$ biotin-E box probe. All binding reactions were performed for $30 \mathrm{~min}$ at room temperature with 0.01 pmol MAX:MAX or $0.4-0.8$ pmol MYC:MAX per reaction. For competition experiments, unlabeled wild-type, or mutant $\mathrm{E}$ box oligos, were added in molar excess over the biotin-E box probe. For SNF5 experiments, SUMO-SNF5, SUMO-SNF5 $\triangle$ RPT, and SUMO-WDR5 were added in molar excess over MYC:MAX or MAX:MAX and incubated for $30 \mathrm{~min}$ at room temperature before the biotin-E-box probe was added. To control for off-target effects of alterations in protein levels, a SUMO-fusion protein encoding amino acids 533-580 of BAF155 was added to maintain the same total molar ratio of protein in each binding reaction. Samples were resolved on a pre-run 5\% native polyacrylamide gel in $0.5 \times$ TBE ( $45 \mathrm{mM}$ Tris-borate, $1 \mathrm{mM}$ EDTA) gel for $25 \mathrm{~min}$ at $150 \mathrm{~V}$ and electroblotted to a nylon membrane (GE Healthcare) for $30 \mathrm{~min}$ at $100 \mathrm{~V}$ in $0.5 \times \mathrm{TBE}$. The membrane was dried and cross-linked by ultraviolet light for 1 min using the optimal crosslink setting on a Spectroline UV Crosslinker Select Series. Detection and visualization of bands was accomplished using the LightShift Chemiluminescent EMSA kit (ThermoFisher Scientific) according to the manufacturer's instructions.

Glycerol sedimentation assay. G401 cells expressing inducible SNF5-HA were plated at $10 \times 10^{6}$ per plate and treated with $1 \mu \mathrm{g} / \mathrm{ml}$ doxycycline for $24 \mathrm{~h}$. Cells were collected and lysed by dounce homogenization in Buffer A (10 mM HEPES (4-(2-hydroxyethyl)-1-piperazineethanesulfonic acid), pH 7.6, $25 \mathrm{mM} \mathrm{KCl}$ $1 \mathrm{mM}$ EDTA, $10 \%$ glycerol, $1 \mathrm{mM}$ dithiothreitol (DTT) with Protease Inhibitor Cocktail; Roche). Nuclei were pelleted at $500 \times g$ for $5 \mathrm{~min}$, resuspended in Buffer B (10 mM HEPES, pH 7.6, $3.5 \mathrm{mM} \mathrm{MgCl}_{2}, 100 \mathrm{mM} \mathrm{KCl}, 0.1 \mathrm{mM}$ EDTA, $10 \%$ glycerol, $1 \mathrm{mM}$ DTT with Protease Inhibitor Cocktail; Roche), and then lysed by addition of ammonium sulfate at a final concentration of $0.3 \mathrm{M}$. Insoluble chromatin fraction was removed by centrifugation $(100,000 \times g)$ for $20 \mathrm{~min}$ in a TLA 100.3 rotor using a tabletop ultracentrifuge (Beckman). To the soluble fraction, 0.3 $\mathrm{mg} / \mathrm{ml}$ ammonium sulfate was added and allowed to incubate for $20 \mathrm{~min}$ on ice. Precipitated proteins were recovered by centrifugation $(100,000 \times g)$ for $30 \mathrm{~min}$ in a TLA 100.3 rotor. Precipitated proteins were resuspended in an HEMG buffer containing no glycerol (25 mM HEPES, pH 7.9, $0.1 \mathrm{mM}$ EDTA, $12.5 \mathrm{mM} \mathrm{MgCl}_{2}$, $100 \mathrm{mM} \mathrm{KCl}, 1 \mathrm{mM} \mathrm{DTT}$ ) and then overlaid onto a $10-\mathrm{ml} \mathrm{10-30 \%} \mathrm{glycerol} \mathrm{gra-}$ dient (in HEMG buffer). Tubes were centrifuged at $4{ }^{\circ} \mathrm{C}$ for $18 \mathrm{~h}$ at $283,000 \times g$. Fractions $(0.5 \mathrm{ml})$ were collected and used to probe proteins by western blot analysis.

Anchorage-independent growth assay. After transduction and selection were complete, 2000 G401 cells expressing TET-inducible EGFP, or SNF5, were mixed with $0.4 \%$ agarose-supplemented DMEM (with TET-approved FBS and $1 \%$ penicillin/streptomycin with $1 \mu \mathrm{g} / \mathrm{ml}$ doxycycline) and added slowly onto a solidified $0.8 \%$ agarose bottom layer. Fresh $1 \mu \mathrm{g} / \mathrm{ml}$ doxycycline was added every $2-3$ days for a total of 14 days. Cells were fixed and stained with $0.05 \%$ crystal violet in $70 \%$ methanol overnight at room temperature and then destained with extensive washing with water. All plates were randomly labeled and analyzed blindly. For A204 cells, the same method was used, except media was replaced once a week for 28 days.

Western blotting and antibodies. G401 cells induced with $1 \mu \mathrm{g} / \mathrm{ml}$ doxycycline for $24 \mathrm{~h}$ (and other cells used in comparing SNF5 levels) were collected in a lysis buffer $(150 \mathrm{mM}$ Tris- $\mathrm{HCl} \mathrm{pH}$ 8.0, $150 \mathrm{mM} \mathrm{NaCl}, 5 \mathrm{mM}$ EDTA, $1 \%$ Triton X-100 with Protease Inhibitor Cocktail; Roche), sonicated at $25 \%$ power for $15 \mathrm{~s}$, and cleared by centrifugation. Protein concentrations were quantified using the Bio-Rad Bradford assay and $10 \mu \mathrm{g}$ of lysate resolved by SDS-PAGE. For HAcoimmunoprecipitation experiments, nuclei from induced cells were extracted in $10 \mathrm{mM}$ HEPES, pH 7.9, $10 \mathrm{mM} \mathrm{KCl}, 0.4 \% \mathrm{NP}-40$ and then lysed in lysis buffer. Equal amounts of nuclear lysates were subjected to immunoprecipitation with $5 \mu \mathrm{l}$ of HA-tag antibody overnight at $4{ }^{\circ} \mathrm{C}$, bound to protein $\mathrm{A}$ agarose (Roche), washed four times in lysis buffer, and resolved by SDS-PAGE. Resolved proteins were transferred to PVDF membrane (PerkinElmer) and blocked in 5\% milk in TBS-T 
(50 mM Tris, pH 7.5, $150 \mathrm{mM} \mathrm{NaCl}, 0.1 \%$ Tween-20). Immunoblotting was per formed using the following antibodies: SNF5 (Bethyl Laboratories, A301-087A, Abcam, ab12167, and Cell Signaling, 91735; all used at 1:1000 dilution), BAF155 (Cell Signaling, D7F8; 1:1000), GAPDH-HRP (Invitrogen, MA5-15738; 1:50,000), HA-epitope tag (Cell Signaling, C29F4; 1:1000), HA-HRP (Roche, 12013819001; 1:2000), MYC (Santa Cruz Biotechnology, sc-274; 1:500; Abcam, Y69; 1:500), and MAX (Santa Cruz Biotechnology, sc-275; 1:500). Visualization of bands were detected using Supersignal West Pico (Pierce).

ChIP and ChIP-Seq. Transduced cells were plated at $10 \times 10^{6}$ per plate and treated with $1 \mu \mathrm{g} / \mathrm{ml}$ doxycycline for $24 \mathrm{~h}$. Cells were crosslinked using $1 \%$ formaldehyde for $10 \mathrm{~min}$, quenched with $0.125 \mathrm{M}$ glycine for $10 \mathrm{~min}$, washed with ice-cold PBS two times, and collected by centrifugation. Nuclei were extracted in $10 \mathrm{mM}$ HEPES, $\mathrm{pH}$ $7.9,10 \mathrm{mM} \mathrm{KCl}, 0.4 \% \mathrm{NP}-40$ and then incubated in $1 \times \mathrm{TE}(10 \mathrm{mM}$ Tris, $\mathrm{pH} 8.0$, $1 \mathrm{mM}$ EDTA) with $1 \%$ SDS for $15 \mathrm{~min}$ on ice. Chromatin was fragmented using sonication with a Diagenode Biorupter, and debris was removed by centrifugation. Chromatin was frozen at $-80^{\circ} \mathrm{C}$ until ready to use. For dTag experiments, HEK293 cells expressing a degradable version of SNF5, cells were plated at $10 \times 10^{6} 4-6$ days post transduction. Then, $500 \mathrm{nM}$ dtag- 47 or matched DMSO control were added for $2 \mathrm{~h}$ and then the above protocol was used to harvest chromatin. Each immunoprecipitation was performed on chromatin collected from $10 \times 10^{6}$ cells by dilution in ten volumes of FALB buffer ( $50 \mathrm{mM}$ HEPES, $\mathrm{pH} 7.5,140 \mathrm{mM} \mathrm{NaCl}, 1 \mathrm{mM}$ EDTA, 1\% Triton) using antibodies against MYC (N262, Santa Cruz Biotechnology, sc-764 or Cell Signaling, 9402; $3 \mu \mathrm{g}$ ) or normal rabbit IgG control (Cell Signaling, $2729 \mathrm{~s} ; 3 \mu \mathrm{g}$ ). Immunoprecipitated DNA was bound to protein A agarose (Roche) and washed sequentially with low salt buffer $(20 \mathrm{mM}$ Tris, $\mathrm{pH} 8.0,150 \mathrm{mM} \mathrm{NaCl}$, $2 \mathrm{mM}$ EDTA, $1 \%$ Triton), high salt buffer ( $20 \mathrm{mM}$ Tris, $\mathrm{pH} 8.0,500 \mathrm{mM} \mathrm{NaCl}, 2$ mM EDTA, 1\% Triton), Lithium Chloride Buffer (10 mM Tris, $\mathrm{pH} 8.0,25 \mathrm{mM} \mathrm{LiCl}$ $1 \mathrm{mM}$ EDTA, $1 \%$ Triton) and two times with $1 \times$ TE. Agarose beads were then resuspended in $1 \times \mathrm{TE}+0.1 \% \mathrm{SDS}+20 \mu \mathrm{g}$ proteinase $\mathrm{K}$ and incubated overnight at $65^{\circ} \mathrm{C}$. For ChIP, samples were diluted in $1 \times \mathrm{TE}$ and coprecipitating DNAs quantified by Q-PCR using the following primers: SNHG15 (CGCCACTGAACCCAATCC and TCTAGTCATCCACCGCCATC), PUM1 (TATGAAGGGACAATCTGCTC and AATCCATCTTCATCCTACCG), CCT7 (TTCCAAAATGATGGTGAGTG and AGAGGGTCCTACAGAGCAAG), METTL1 (GCATGGCTGCGTCATTAACT and GAGTCTCGGCTGCCATGAT), RPS24 (TTGGCTGTCTGAAGATAGATCG and CGCGTGCCTATAGCTCAAGT), RPL5 (CCTGCAGGTCTCTGTCGAG and GGCATACGGGCAAGAAAAG), RPL35 (CTTGTGCAGCAATGGTGAGA and GCCTAGGTGGCAGATAGAATC), RPL10 (GCAAGAGTTCTACGCCCAAG and CACATGCGCAGATCAGAGAG), RNSP1 (GATGTAAGTTGGGGCGGAAT and GAGGAGTGGACCGGCTTC), and SNHG15 GB (AATTATGTGTCCAGGGTT GC and CACCGGCTTCTATATTCCAC). For ChIP-Seq, DNA from the equivalent of $30 \times 10^{6}$ cells was combined and purified using a Qiagen PCR purification kit, and eluted DNA was used to generate libraries. Libraries were made using NEBnext Ultra II DNA library Prep protocol and NEBNext Multiplex Oligos for Illumina, with the addition of an AMPure clean-up step prior to beginning end repair. Sequencing data were obtained on an Illumina NextSeq500 with 75 bp single reads. Sequencing was performed by the VANTAGE Core at Vanderbilt University.

ATAC-Seq. Cells were plated at $1 \times 10^{6}$ per plate with $1 \mu \mathrm{g} / \mathrm{ml}$ doxycycline for $24 \mathrm{~h}$ and then 75,000 cells were harvested following published protocols ${ }^{34,49}$. Briefly, cells were harvested and nuclei were extracted in $10 \mathrm{mM}$ Tris- $\mathrm{HCl}, \mathrm{pH} 7.4,10 \mathrm{mM}$ $\mathrm{NaCl}, 3 \mathrm{mM} \mathrm{MgCl}, 0.1 \%$ IGEPAL CA-630 (Sigma). Nuclei were immediately added to the transposase reaction containing Tn5 Transposase (Illumina Nextera DNA Kit) for $30 \mathrm{~min}$ at $37^{\circ} \mathrm{C}$. Reactions were stopped by purification with the Qiagen PCR Purification Kit. Transposed DNA was used to generate libraries through PCR amplification with NEBNext High-Fidelity $2 \times$ PCR Master Mix and Nextera-based primers (obtained from IDT). Amplified libraries were submitted to Genewiz for sequencing on an Illumina HiSeq2500 with 50 bp paired end reads.

PRO-Seq. Cells were plated at $10 \times 10^{6}$ per plate with $1 \mu \mathrm{g} / \mathrm{ml}$ doxycycline for $24 \mathrm{~h}$ and then $30 \times 10^{6}$ cells were combined per condition and harvested together according to published protocols ${ }^{50}$, with minor changes. Briefly, nuclei were extracted in $10 \mathrm{mM}$ Tris- $\mathrm{HCl}, \mathrm{pH} 7.4,300 \mathrm{mM}$ sucrose, $10 \mathrm{mM} \mathrm{KCl}, 5 \mathrm{mM} \mathrm{MgCl}_{2}$, $1 \mathrm{mM}$ EGTA, $0.05 \%$ Tween, $0.1 \%$ NP-40, $0.5 \mathrm{mM}$ DTT, RNAse inhibitor and protease inhibitor cocktail (Roche) and then snap-frozen in $10 \mathrm{mM}$ Tris- $\mathrm{HCl}, \mathrm{pH}$ $8.0,25 \%$ glycerol, $5 \mathrm{mM} \mathrm{MgCl} 2,0.1 \mathrm{mM}$ EDTA, $5 \mathrm{mM}$ DTT with protease inhibitor cocktail and stored at $-80^{\circ} \mathrm{C}$ until ready to use. Biotin run-on reactions were performed on thawed nuclei in a reaction buffer containing Biotin-11-CTP (PerkinElmer, NEL542001) for $3 \mathrm{~min}$ at $30^{\circ} \mathrm{C}$. Reactions were stopped by adding Trizol LS (Thermo Scientific) and RNA purified by chloroform and isopropanol extraction. Resuspended RNA pellets were heated at $65^{\circ} \mathrm{C}$ for $40 \mathrm{~s}$ and $1 \mathrm{M} \mathrm{NaOH}$ was added, followed by incubation on ice for $10 \mathrm{~min}$. Base hydrolysis was neutralized by addition of $1 \mathrm{M}$ Tris ( $\mathrm{pH}$ 6.8) and the sample ran over a Micro Bio-Spin P-30 gel column (Bio-Rad). Strepavidin Dynabeads (ThermoFisher, 65601) were incubated with collected material and biotinylated RNA bound to beads using their standard protocol. Elution of bound, biotinylated, RNA was achieved by extracting beads with Trizol and subsequently purified using chloroform and isopropanol. RNA adaptors (IDT) were added to the $3^{\prime}$ side biotinylated RNA and following a second round of biotin-RNA purification, $5^{\prime}$ RNA caps were removed using CAP CLIP (CellScript, C-CC15011H). 5' RNA adaptors (IDT) were then added. One additional biotin-RNA purification was performed, and purified RNA was used in a reverse transcriptase reaction to generate cDNA. Libraries were amplified using the generated cDNA and a PCR cycle number determined from a test analysis of a portion of sample. Library amplification was performed with Phusion high-fidelity polymerase (NEB) and customized Illumina-based index primers (IDT). Sequencing data were obtained on an Illumina NextSeq500 with $75 \mathrm{bp}$ single reads. Sequencing was performed by the VANTAGE Core at Vanderbilt University.

ChIP-Seq processing and analysis. ChIP-seq reads were aligned to the human genome using Bowtie ${ }^{51}$. Peaks in each sample were called using MACS2 with $q$ value of $0.01^{52}$. Peaks were annotated using Homer command annotatePeaks, and enriched motifs were identified by Homer command findMotifsGenome with the default region size and the motif length (-size 200 and -len 8, 10, 12) (http://homer ucsd.edu/homer/). Consensus peaks in each condition were identified using DiffBind $^{53}$, where peaks occurring both replicates were included. Peaks identified across conditions were combined into a final peak set and ChIP read counts were calculated for the final peak set. Read counts were normalized to the total mapped reads, and differential peaks were determined by DESeq $2^{54}$, which calculated the $\log _{2}$ fold changes, Wald test $p$ values, and adjusted $p$ values (False Discovery Rate, FDR) by the Benjamini-Hochberg procedure. The significantly changed peaks were assessed with an FDR $<0.05$. Hypergeometric test was used to estimate the enrichment of MYC target genes in MSigDB Hallmark data sets using all human genes as background. The overlap of EGFP MYC peaks with published MYC peaks was determined by DiffBind ${ }^{53}$ with default parameters. The heatmaps were generated by the average normalized peak intensity within $\pm 2 \mathrm{~kb}$ from peak center with $100 \mathrm{bp}$ bin size. The peak read graph showed the average normalized peak intensity in EGFP, SNF5 and OMOMYC, where peaks were ranked by the normalized intensity in EGFP. GO term analysis was performed on genes assigned to MYC peaks that fell within $1 \mathrm{~kb}$ of promoter using functional annotation clustering through DAVID (https://david.ncifcrf.gov/). Correlation of ChIP-Seq replicates on normalized counts of all promoters is presented in Supplementary Fig. 8a.

ATAC-Seq processing and analysis. Adapter sequences of ATAC-seq reads were trimmed by cutadapt ${ }^{55}$ (cutadapt -a CTGTCTCTTATACACATCT-minimumlength 15 -paired-output), then aligned to the human genome using Bowtie $2^{51}$ (bowtie2 -p 8 -X 2000 -q-no-mixed-no-discordant). Peaks in each sample were called using MACS2 with a $q$ value of 0.001 (callpeak -q 0.001 -nomodel -extsize $140)^{52}$. Peaks were annotated using Homer command annotatePeaks to determine whether peaks were near TSS promoter or far away from TSS (TSS-distal). Enriched motifs were identified by Homer command findMotifsGenome with the default region size and the motif length (-size 200 and -len 8, 10, 12) (http://homer. ucsd.edu/homer/). Consensus peaks in each condition were identified by DiffBind ${ }^{53}$ where peaks occurring at least two replicates were included. Peaks identified across conditions were combined into a final peak set and ATAC-seq read counts for the final peak set were calculated using DiffBind ${ }^{53}$. Read counts were normalized by the RLE method, and differential peaks were identified by DESeq2 ${ }^{54}$. The significantly changed peaks were assessed with an FDR $<0.05$. GO term analysis was performed on annotated genes (HOMER) that were assigned to gained ATAC-seq peaks following SNF5 reintroduction using functional annotation clustering through DAVID (https://david.ncifcrf.gov/). Correlation of ATAC-Seq replicates on normalized counts of all promoters is presented in Supplementary Fig. 8b.

PRO-Seq processing and analysis. After adapter trimming and low-quality sequence removal by cutadapt ${ }^{55}$, PRO-seq reads longer than $15 \mathrm{bp}$ were reversed complemented using FastX tools ${ }^{8}$. Reversed complemented reads were aligned to human genome using Bowtie $2^{51}$. Reads mapped to rRNA loci and reads with mapping quality less than 10 were removed. The reads were normalized by the RLE implemented in the DESeq2 ${ }^{54}$. NRSA (http://bioinfo.vanderbilt.edu/NRSA/), a tool to provide a comprehensive analysis on nascent transcriptional profiles for known genes, was used to estimate RNA polymerase abundance in proximalpromoter and gene body regions of genes, to calculate pausing index and pausing index alterations. Briefly, the promoter-proximal region is defined by examining each $50 \mathrm{bp}$ window with a $5 \mathrm{bp}$ sliding step along the coding strand spanning \pm $500 \mathrm{bp}$ from known TSSs. The $50 \mathrm{bp}$ region with the largest number of reads is considered as the promoter-proximal region and its read density is calculated ${ }^{56}$. Gene body is defined as the region from $+1 \mathrm{~kb}$ downstream of a TSS to its transcription termination site (TTS). Pausing index for each gene is calculated as the ratio of promoter-proximal density over gene body density and the significance of pausing is evaluated by Fisher's exact test ${ }^{56}$. DESeq $2^{54}$ was implemented to detect significant transcriptional changes for promoter-proximal and gene body regions accounting for the batch effect. The significantly transcriptional changes were assessed with an FDR $<0.05$ or $<0.0001$ as described in figure legends. GO term analysis was performed on the overlapped set of genes with an increased pausing index between SNF5 and OMOMYC using functional annotation clustering through DAVID (https://david.ncifcrf.gov/). Correlation of PRO-Seq replicates on gene body densities is presented in Supplementary Fig. 8c. 
Reporting summary. Further information on research design is available in the Nature Research Reporting Summary linked to this article.

\section{Data availability}

All sequencing data have been deposited at GEO with the accession number GSE109310. Routine metrics for all next-generation sequencing data are presented in Supplementary Table 2. Any other data supporting the findings in this study are available upon request. Uncropped scans for all blots are presented in Supplementary Fig. 9.

Received: 2 February 2018 Accepted: 12 April 2019

Published online: 01 May 2019

\section{References}

1. Arnaud, O., Le Loarer, F. \& Tirode, F. BAFfling pathologies: alterations of BAF complexes in cancer. Cancer Lett. 419, 266-279 (2018).

2. Kadoch, C. et al. Proteomic and bioinformatic analysis of mammalian SWI/ SNF complexes identifies extensive roles in human malignancy. Nat. Genet. 45, 592-601 (2013)

3. Kadoch, C. \& Crabtree, G. R. Reversible disruption of mSWI/SNF (BAF) complexes by the SS18-SSX oncogenic fusion in synovial sarcoma. Cell 153, 71-85 (2013).

4. Savas, S. \& Skardasi, G. The SWI/SNF complex subunit genes: their functions, variations, and links to risk and survival outcomes in human cancers. Crit. Rev. Oncol. Hematol. 123, 114-131 (2018).

5. Wang, W. et al. Purification and biochemical heterogeneity of the mammalian SWI-SNF complex. EMBO J. 15, 5370-5382 (1996).

6. Roberts, C. W., Galusha, S. A., McMenamin, M. E., Fletcher, C. D. \& Orkin, S. H. Haploinsufficiency of Snf5 (integrase interactor 1) predisposes to malignant rhabdoid tumors in mice. Proc. Natl Acad. Sci. USA 97, 13796-13800 (2000).

7. Roberts, C. W., Leroux, M. M., Fleming, M. D. \& Orkin, S. H. Highly penetrant, rapid tumorigenesis through conditional inversion of the tumor suppressor gene Snf5. Cancer Cell 2, 415-425 (2002).

8. Gadd, S., Sredni, S. T., Huang, C. C., Perlman, E. J. \& Renal Tumor Committee of the Children's Oncology, G. Rhabdoid tumor: gene expression clues to pathogenesis and potential therapeutic targets. Lab. Invest. 90, 724-738 (2010)..

9. Wang, X. et al. TCR-dependent transformation of mature memory phenotype T cells in mice. J. Clin. Investig. 121, 3834-3845 (2011).

10. Genovese, G. et al. Synthetic vulnerabilities of mesenchymal subpopulations in pancreatic cancer. Nature 542, 362-366 (2017)

11. Lee, R. S. et al. A remarkably simple genome underlies highly malignant pediatric rhabdoid cancers. J. Clin. Investig. 122, 2983-2988 (2012).

12. Wang, X. et al. SMARCB1-mediated SWI/SNF complex function is essential for enhancer regulation. Nat. Genet. 49, 289-295 (2017).

13. Ae, K. et al. Chromatin remodeling factor encoded by inil induces G1 arrest and apoptosis in ini1-deficient cells. Oncogene 21, 3112-3120 (2002).

14. Betz, B. L., Strobeck, M. W., Reisman, D. N., Knudsen, E. S. \& Weissman, B. E. Re-expression of hSNF5/INI1/BAF47 in pediatric tumor cells leads to G1 arrest associated with induction of pl6ink4a and activation of RB. Oncogene 21, 5193-5203 (2002).

15. Versteege, I., Medjkane, S., Rouillard, D. \& Delattre, O. A key role of the hSNF5/INI1 tumour suppressor in the control of the G1-S transition of the cell cycle. Oncogene 21, 6403-6412 (2002).

16. Zhang, Z. K. et al. Cell cycle arrest and repression of cyclin D1 transcription by INI1/hSNF5. Mol. Cell Biol. 22, 5975-5988 (2002).

17. Oruetxebarria, I. et al. P16INK4a is required for hSNF5 chromatin remodelerinduced cellular senescence in malignant rhabdoid tumor cells. J. Biol. Chem. 279, 3807-3816 (2004)

18. Vries, R. G. et al. Cancer-associated mutations in chromatin remodeler hSNF5 promote chromosomal instability by compromising the mitotic checkpoint. Genes Dev. 19, 665-670 (2005).

19. Cheng, S. W. et al. c-MYC interacts with INI1/hSNF5 and requires the SWI/ SNF complex for transactivation function. Nat. Genet. 22, 102-105 (1999).

20. Takayama, M. A., Taira, T., Tamai, K., Iguchi-Ariga, S. M. \& Ariga, H. ORC1 interacts with c-Myc to inhibit E-box-dependent transcription by abrogating c-Myc-SNF5/INI1 interaction. Genes Cells 5, 481-490 (2000).

21. Stojanova, A. et al. MYC interaction with the tumor suppressive SWI/SNF complex member INI1 regulates transcription and cellular transformation. Cell Cycle 15, 1693-1705 (2016).

22. Tansey, W. P. Mammalian MYC proteins and cancer. New J. Sci. 2013, 1-27 (2014).

23. Alimova, I. et al. Inhibition of MYC attenuates tumor cell self-renewal and promotes senescence in SMARCB1 deficient Group 2 Atypical Teratoid Rhabdoid Tumors to suppress tumor growth in vivo. Int. J. Cancer 144, 1983-1995 (2019).
24. Farina, A., Faiola, F. \& Martinez, E. Reconstitution of an E box-binding Myc: Max complex with recombinant full-length proteins expressed in Escherichia coli. Protein Expr. Purif. 34, 215-222 (2004)

25. Thomas, L. R. et al. Interaction with WDR5 promotes target gene recognition and tumorigenesis by MYC. Mol. Cell 58, 440-452 (2015).

26. Nabet, B. et al. The dTAG system for immediate and target-specific protein degradation. Nat. Chem. Biol. 14, 431-441 (2018).

27. Huang, H. T. et al. MELK is not necessary for the proliferation of basal-like breast cancer cells. eLife 6, https://doi.org/10.7554/eLife.26693 (2017).

28. Thomas, L. R. et al. Interaction of MYC with Host Cell Factor-1 is mediated by the evolutionarily-conserved Myc box IV motif. Oncogene 35, 3613-3618 (2105).

29. Soucek, L. et al. Design and properties of a Myc derivative that efficiently homodimerizes. Oncogene 17, 2463-2472 (1998)

30. Soucek, L. et al. Omomyc, a potential Myc dominant negative, enhances Mycinduced apoptosis. Cancer Res. 62, 3507-3510 (2002).

31. Savino, M. et al. The action mechanism of the Myc inhibitor termed Omomyc may give clues on how to target Myc for cancer therapy. PLOS ONE 6, e22284 (2011).

32. Jung, L. A. et al. OmoMYC blunts promoter invasion by oncogenic MYC to inhibit gene expression characteristic of MYC-dependent tumors. Oncogene 36, 1911-1924 (2017).

33. Thomas, L. R. \& Tansey, W. P. MYC and chromatin. Open Access J. Sci. Technol. 3, 101124 (2015).

34. Buenrostro, J. D., Giresi, P. G., Zaba, L. C., Chang, H. Y. \& Greenleaf, W. J. Transposition of native chromatin for fast and sensitive epigenomic profiling of open chromatin, DNA-binding proteins and nucleosome position. Nat. Methods 10, 1213-1218 (2013).

35. Vierbuchen, T. et al. AP-1 transcription factors and the BAF complex mediate signal-dependent enhancer selection. Mol. Cell 68, 1067-1082 (2017).

36. Alver, B. H. et al. The SWI/SNF chromatin remodelling complex is required for maintenance of lineage specific enhancers. Nat. Commun. 8, 14648 (2017).

37. Nakayama, R. T. et al. SMARCB1 is required for widespread BAF complexmediated activation of enhancers and bivalent promoters. Nat. Genet. 49, 1613-1623 (2017)

38. Rahl, P. B. et al. c-Myc regulates transcriptional pause release. Cell 141, 432-445 (2010)

39. Kwak, H., Fuda, N. J., Core, L. J. \& Lis, J. T. Precise maps of RNA polymerase reveal how promoters direct initiation and pausing. Science 339, 950-953 (2013).

40. Zhao, Y. et al. High-resolution mapping of RNA polymerases identifies mechanisms of sensitivity and resistance to BET inhibitors in t(8;21) AML. Cell Rep. 16, 2003-2016 (2016).

41. Pawel, B. R. SMARCB1-deficient tumors of childhood: a practical guide. Pediatr. Dev. Pathol. 21, 6-28 (2018).

42. Sammak, S., Allen, M. D., Hamdani, N., Bycroft, M. \& Zinzalla, G. he structure of INI1/hSNF5 RPT1 and its interactions with the c-MYC:MAX heterodimer provide insights into the interplay between MYC and the SWI/SNF chromatin remodeling complex. FEBS J. 285, 4165-4180 (2018).

43. You, J. S. et al. SNF5 is an essential executor of epigenetic regulation during differentiation. PLoS Genet. 9, e1003459 (2013).

44. Sabo, A. \& Amati, B. Genome recognition by MYC. Cold Spring Harbor Perspectives in Medicine 4, https://doi.org/10.1101/cshperspect.a014191 (2014).

45. Fruhwald, M. C., Biegel, J. A., Bourdeaut, F., Roberts, C. W. \& Chi, S. N. Atypical teratoid/rhabdoid tumors-current concepts, advances in biology, and potential future therapies. Neuro. Oncol. https://doi.org/10.1093/neuonc/ nov264 (2016).

46. Whitfield, J. R., Beaulieu, M. E. \& Soucek, L. Strategies to inhibit Myc and their clinical applicability. Front Cell Dev. Biol. 5, 10 (2017).

47. Campeau, E. et al. A versatile viral system for expression and depletion of proteins in mammalian cells. PLoS ONE 4, e6529 (2009).

48. Meerbrey, K. L. et al. The pINDUCER lentiviral toolkit for inducible RNA interference in vitro and in vivo. Proc. Natl Acad. Sci. USA 108, 3665-3670 (2011).

49. Buenrostro, J. D., Wu, B., Chang, H. Y. \& Greenleaf, W. J. ATAC-seq: a method for assaying chromatin accessibility genome-wide. Curr. Protoc. Mol. Biol. 109, 21-29 (2015)

50. Mahat, D. B. et al. Base-pair-resolution genome-wide mapping of active RNA polymerases using precision nuclear run-on (PRO-seq). Nat. Protoc. 11, 1455-1476 (2016)

51. Langmead, B., Trapnell, C., Pop, M. \& Salzberg, S. L. Ultrafast and memoryefficient alignment of short DNA sequences to the human genome. Genome Biol. 10, R25 (2009).

52. Feng, J., Liu, T., Qin, B., Zhang, Y. \& Liu, X. S. Identifying ChIP-seq enrichment using MACS. Nat. Protoc. 7, 1728-1740 (2012). 
53. Stark, R. \& Brown, G. DiffBind: differential binding analysis of ChIP-seq peak data. Bioconductor http://bioconductor.org/packages/release/bioc/vignettes/ DiffBind/inst/doc/DiffBind.pdf (2011).

54. Love, M. I., Huber, W. \& Anders, S. Moderated estimation of fold change and dispersion for RNA-seq data with DESeq2. Genome Biol. 15, 550 (2014).

55. Martin, M. Cutadapt removes adapter sequences from high-throughput sequencing reads. EMBnet J. 17, 10-12 (2011).

56. Core, L. J., Waterfall, J. J. \& Lis, J. T. Nascent RNA sequencing reveals widespread pausing and divergent initiation at human promoters. Science 322, 1845-1848 (2008).

\section{Acknowledgements}

We thank Scott Hiebert for his critical contributions to the study and the Vanderbilt University Chemical Synthesis Core for synthesis of the dTag47 molecule. This work was supported by grants from St. Baldrick's Foundation, Alex's Lemonade Stand Foundation, the Edward P. Evans Foundation, the Robert J. Kleberg, Jr. and Helen C. Kleberg Foundation, and grant CA200709 from the National Institutes of Health (to W.P.T.); The Rally Foundation for Childhood Cancer Research Fellowship, Open Hands Overflowing Hearts co-funded Research Fellowship, and the American Association for Cancer Research Basic Cancer Research Fellowship to A.M.W.; The Vanderbilt Ingram Cancer Center Support grant (NIH: CA68485); and by CTSA award UL1TR002243 from the National Center for Advancing Translational Sciences.

\section{Author contributions}

A.M.W. and W.P.T. designed the experiments, interpreted the data, and wrote the manuscript. A.M.W. executed the experiments. A.M.W., S.L.L. and G.C.H. performed analyses of data. J.W. and Q.L. performed and oversaw all computational and statistical analyses. E.M. contributed to experimental design and provided key reagents.

\section{Additional information}

Supplementary Information accompanies this paper at https://doi.org/10.1038/s41467019-10022-5.

Competing interests: The authors declare no competing interests.

Reprints and permission information is available online at http://npg.nature.com/ reprintsandpermissions/

Journal peer review information: Nature Communications thanks Hojoong Kwak, Christian Muchardt and other anonymous reviewer(s) for their contribution to the peer review of this work.

Publisher's note: Springer Nature remains neutral with regard to jurisdictional claims in published maps and institutional affiliations.

(c) (i) Open Access This article is licensed under a Creative Commons Attribution 4.0 International License, which permits use, sharing, adaptation, distribution and reproduction in any medium or format, as long as you give appropriate credit to the original author(s) and the source, provide a link to the Creative Commons license, and indicate if changes were made. The images or other third party material in this article are included in the article's Creative Commons license, unless indicated otherwise in a credit line to the material. If material is not included in the article's Creative Commons license and your intended use is not permitted by statutory regulation or exceeds the permitted use, you will need to obtain permission directly from the copyright holder. To view a copy of this license, visit http://creativecommons.org/ licenses/by/4.0/.

(C) The Author(s) 2019 